\title{
Indicadores de desenvolvimento sustentável nos municípios do Nordeste Paraense
}

O desenvolvimento sustentável constitui-se no novo paradigma de desenvolvimento vigente, por isso se faz necessário a utilização de métodos de avaliação de sustentabilidade para servir de subsídios na formulação de políticas públicas. Neste artigo avalia-se os níveis de sustentabilidade dos municípios da mesorregião do Nordeste Paraense, no estado do Pará, mediante o cálculo do Índice de Desenvolvimento Sustentável para Municípios (IDSM). A metodologia se baseou na utilizada por Martins e Cândido (2012), onde os dados são analisados de acordo com seis dimensões (social, demográfica, econômica, político-institucional, ambiental e cultural) e quatro níveis (ideal, aceitável, alerta e crítico), a partir de dados secundários obtidos nas bases de dados do Atlas do Desenvolvimento Humano no Brasil, FAPESPA, DATASUS, IBGE, FINBRA, ANATEL, TSE e SNIS. Os resultados foram ilustrados através de mapas visando uma melhor representação da realidade dos municípios estudados. A pesquisa constatou que $94 \%$ dos municípios analisados foram classificados no nível de alerta, demonstrando a necessidade de políticas públicas mais adequadas ao favorecimento do desenvolvimento sustentável. A metodologia utilizada se mostrou eficiente para orientar os gestores públicos em suas tomadas de decisões, principalmente no que diz respeito à qualidade da educação, oferta de serviços básicos de saúde, saneamento básico, incentivo ao setor industrial e a construção de equipamentos culturais como museus, teatros e bibliotecas.

Palavras-chave: Cidades da Amazônia; Desenvolvimento Regional; Gestão Pública; Índice de Desenvolvimento Sustentável para Municípios; Sustentabilidade.

\section{Sustainable development indicators in the municipalities of Northeast Paraense}

\begin{abstract}
Sustainable development constitutes the new development paradigm in force, which is why it is necessary to use methods of sustainability assessment to serve as subsidies in the formulation of public policies. In this article, the sustainability levels of the municipalities in the mesoregion of Northeast Paraense, in the state of Pará, are evaluated by calculating the Sustainable Development Index for Municipalities (IDSM). The methodology was based on that used by Martins and Cândido (2012), where the data are analyzed according to six dimensions (social, demographic, economic, political-institutional, environmental and cultural) and four levels (ideal, acceptable, alert and critical), from secondary data obtained in the databases of the Atlas of Human Development in Brazil, FAPESPA, DATASUS, IBGE, FINBRA, ANATEL, TSE and SNIS. The results were illustrated through maps aiming at a better representation of the reality of the studied cities. The survey found that $94 \%$ of the municipalities analyzed were classified at the level of alert, demonstrating the need for more appropriate public policies to favor sustainable development. The methodology used proved to be efficient in guiding public managers in their decision making, especially with regard to the quality of education, the provision of basic health services, basic sanitation, incentives to the industrial sector and the construction of cultural facilities such as museums, theaters and libraries.
\end{abstract}

Keywords: Cities in the Amazon; Regional development; Public Management; Sustainable Development Index for Municipalities; Sustainability.

Topic: Desenvolvimento, Sustentabilidade e Meio Ambiente

Reviewed anonymously in the process of blind peer.
Received: $10 / 08 / 2020$

Approved: 21/09/2020
Caio Cezar Ferreira de Souza (iD)

Universidade da Amazônia, Brasil

http://lattes.cnpq.br/9164780363813621

http://orcid.org/0000-0002-0038-277X

caiocfdesouza@gmail.com

Ellen Gleyce da Silva Lima (it)

Universidade Federal Rural da Amazônia, Brasil

http://lattes.cnpq.br/3608078786094756

http://orcid.org/0000-0002-3296-6243

ellenllima@yahoo.com.br

Marcos Antônio Souza dos Santos (iD)

Universidade Federal Rural da Amazônia, Brasil

http://lattes.cnpq.br/1517009704490133

http://orcid.org/0000-0003-1028-1515

marcos.marituba@gmail.com

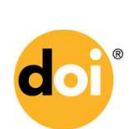

DOI: 10.6008/CBPC2179-6858.2020.005.0041

\author{
Fabrício Khoury Rebello (iD \\ Universidade Federal Rural da Amazônia, Brasil \\ http://lattes.cnpq.br/6299203737952237 \\ http://orcid.org/0000-0003-2398-4906 \\ fabriciorebello@hotmail.com \\ Cyntia Meireles Martins (it) \\ Universidade Federal Rural da Amazônia, Brasil \\ http://lattes.cnpq.br/6299203737952237 \\ http://orcid.org/0000-0002-5695-8504 \\ cyntiamei@hotmail.com \\ Maria Lúcia Bahia Lopes (iD) \\ Universidade da Amazônia, Brasil \\ http://lattes.cnpq.br/4629419656109116 \\ http://orcid.org/0000-0002-3718-0434 \\ marialucia.bahia@unama.br
}

Referencing this:

SOUZA, C. C. F.; LIMA, E. G. S.; SANTOS, M. A. S.; REBELLO, F. K.; MARTINS, C. M.; LOPES, M. L. B.. Indicadores de desenvolvimento sustentável nos municípios do Nordeste Paraense. Revista Ibero Americana de Ciências Ambientais, v.11, n.5, p.451-462, 2020. DOI: http://doi.org/10.6008/CBPC2179-6858.2020.005.0041 


\section{INTRODUÇÃO}

A busca por desenvolvimento sustentável como projeto político e social da humanidade tem promovido a orientação de esforços no sentido de encontrar caminhos para sociedades sustentáveis (SALASZAPATA et al., 2011). Desde então, surge grande quantidade de literatura dedicada ao tema, e sem dúvida uma indefinição de foco.

Sustentabilidade é o equilíbrio entre os três pilares: ambiental, econômico e social (ELKINGTON, 1994). A sustentabilidade ambiental é definida como a desmaterialização da atividade econômica, pois uma diminuição do processamento de material pode reduzir a pressão sobre os sistemas naturais e ampliar a prestação de serviços ambientais para a economia. A sustentabilidade econômica é a manutenção de capital natural, que é uma condição necessária para não haver decrescimento econômico (BARTELMUS, 2003). A abordagem da sustentabilidade social, por sua vez, refere-se à homogeneidade social, rendimentos justos e acesso a bens, serviços e emprego (LEHTONEN, 2004).

A preocupação com a sustentabilidade não surge de agora, no final do século 1920 a sociedade começou a perceber os impactos da ação humana no meio ambiente. Foi então que ganharam forças políticas de desenvolvimento com foco na sustentabilidade. Essas políticas, no entanto, foram implementadas de maneira pouco eficaz ao longo dos anos, e até mesmo a conceituação de desenvolvimento sustentável é divergente para boa parte dos que se debruçam sobre o tema (REZENDE et al., 2017).

Rezende et al. (2017) mencionam que, para contribuir na gestão dos recursos naturais, foram definidos indicadores de sustentabilidade, que ao examinar fatos e políticas públicas mediante a comparação de cidades e regiões, contribuíram para a avaliação do grau de sustentabilidade do desenvolvimento da sociedade, sinalizando quais índices são positivos e quais necessitam de medidas proativas e reativas a serem adotadas, antes que danos irreversíveis possam ocorrer. Indicadores são cruciais para guiar os tomadores de decisão em uma variedade de caminhos de condução de políticas públicas, pois as informações geradas por eles facilitam o processo de decisão e podem ajudar a mensurar o desempenho de políticas voltadas para o desenvolvimento sustentável.

Os indicadores são ferramentas que possibilitam a obtenção de dados ou informações sobre uma dada realidade espacial, ou que procura descrever um processo específico ou um processo de controle (MASERA et al., 1999). Um indicador pode ser quantitativo e qualitativo, não sendo restrito a apenas uma dessas esferas. Um indicador não é apenas uma estatística, ele representa uma variável que assume um valor em um tempo específico. Por sua vez, uma variável é a representação de um atributo de um determinado sistema, incluindo qualidade, característica e propriedade (QUIROGA, 2001). No contexto do desenvolvimento sustentável, um conjunto de indicadores de sustentabilidade exerce a função de advertir a comunidade sobre riscos e tendências do desenvolvimento, constituindo-se como uma carta de navegação sobre o futuro (GUIMARÃES, 1998).

No contexto municipal, a utilização dos indicadores pelos gestores públicos é extremamente relevante para demonstrar as intervenções necessárias a fim de corrigir discrepâncias encontradas, 
viabilizando o desenvolvimento sustentável local, e consequentemente regional, atingindo então um grau de sustentabilidade efetiva (REZENDE et al., 2017).

A metodologia desenvolvida por Martins et al. (2012) é capaz de aferir o índice de desenvolvimento sustentável de determinado município. Essa metodologia é integrada pelo conjunto de seis dimensões (social, demográfica, ambiental, econômica, político-institucional e cultural), cada qual com seus respectivos indicadores.

Partindo desses pressupostos, o presente artigo tem como finalidade a utilização do Índice de Desenvolvimento Sustentável para Municípios (IDSM), com o propósito da avaliação da sustentabilidade dos municípios no Nordeste Paraense. Assim, as variáveis coletadas, tratadas e analisadas neste estudo, permitiram estimar um indicador de sustentabilidade municipal, organizado por dimensões, que permite aferir os níveis de sustentabilidade das localidades investigadas.

\section{REVISÃO TEÓRICA}

\section{Desenvolvimento e Sustentabilidade}

O termo Desenvolvimento Sustentável surgiu da necessidade de se repensar as formas de apropriação do meio natural pelo homem, assim como de elaborar novas estratégias produtivas na tentativa de minimizar os custos socioambientais ocasionados pelo atual modelo produtivo. O Relatório Brundtland define desenvolvimento sustentável como:

Aquele que responde às necessidades do presente sem comprometer a capacidade das gerações futuras em atender às suas próprias necessidades.

[...] um processo de mudança no qual a exploração, a direção de investimentos, a orientação do desenvolvimento tecnológico e, mudanças institucionais estão todas em harmonia e ambos aumentam o potencial corrente e futuro para reunir necessidades e aspirações humanas. (CMMAD, 1991)

Segundo Dahl (1997) é um conceito carregado de valores, e existe uma forte relação entre os princípios, a ética, as crenças e os valores que fundamentam uma sociedade ou comunidade e sua concepção de sustentabilidade. Pondera que um dos problemas do conceito se refere ao fato de que a sociedade deve saber para onde quer ir para que depois se possa medir se esses objetivos ou direção estão sendo seguidos ou alcançados. Para alcançar o desenvolvimento sustentável deve-se chegar a uma concepção que seja compreensiva e, ao mesmo tempo, compreensível do conceito. Ou seja, que consiga captar o conceito de desenvolvimento sustentável ao mesmo tempo em que transmite essa concepção para os atores da sociedade de uma maneira mais clara.

De acordo com Ayres (2008), a sustentabilidade é um conceito normativo sobre a maneira como os seres humanos devem agir em relação à natureza, e como eles são responsáveis para com o outro e as futuras gerações. Neste contexto, observa-se que a sustentabilidade é condizente ao crescimento econômico baseado na justiça social e eficiência no uso de recursos naturais (LOZANO, 2012). 


\section{Indicadores}

Indicadores são usados para monitorar sistemas complexos, são sinais de eventos, são informações que apontam as características ou o que está ocorrendo com o sistema podendo ser uma variável ou uma função de variáveis (SIENA, 2002). Um indicador ajuda a compreender onde se está, qual o caminho a ser seguido e a que distância se está da meta estabelecida. Ajuda a identificar os problemas antes que se tornem insuperáveis e auxiliam na sua solução. Para que um indicador seja efetivo é necessário que seja relevante, refletindo o sistema que precisa ser conhecido, fácil de ser entendido, confiável e baseado em dados acessíveis (HART, 1999).

A mais importante característica do indicador, quando comparado com os outros tipos ou formas de informação, é a sua relevância para a política e para o processo de tomada de decisão. Para ser representativo, o indicador tem de ser considerado importante tanto pelos tomadores de decisão quanto pelos diversos agentes relacionados ao processo (GALLOPIN, 1996).

Indicadores são ferramentas que proporcionam informar a cerca de determinada condição, fenômeno ou tendência, tornando a comunicação acerca destes, mais compreensível e quantificável, de acordo também com seu próprio termo originário do Latim (indicare - apontar, indicar algo) (BELLEN, 2006). Moura et al. (2004) definem indicador de sustentabilidade como um conjunto de parâmetros que permita medir as modificações antrópicas em um determinado sistema e comunicar, de forma simplificada, o estado deste sistema em relação aos critérios e as metas estabelecidas para avaliar a sua sustentabilidade.

\section{Importância do uso de índices de desempenho para mensurar o desenvolvimento sustentável}

As dimensões econômicas, sociais, ambientais e institucionais do desenvolvimento sustentável são complexas e envolvem sinergias e relacionamentos de influência mútua. A lista de indicadores capazes de capturar e avaliar a orientação de sustentabilidade é influenciada pela interdependência nestas dimensões. Kates et al. (2001) defendem que o objetivo da mensuração da sustentabilidade é fornecer, aos tomadores de decisão, os elementos de avaliação nos níveis global e local. Essa mensuração deve integrar os sistemas naturais com a sociedade, e auxiliar nas decisões de curto e longo prazo. De acordo com Segnestam (2002), indicadores são usados como ferramenta para obter informações sobre questões que envolvem saúde populacional, clima e bem-estar econômico.

Cornescu et al. (2014) argumentam que o uso de indicadores permite apenas uma visão parcial do desenvolvimento sustentável e envolve temas específicos. Esses temas incluem as necessidades humanas (i.e., saúde, alimentação, habitação, educação, equidade e segurança), a economia (i.e., padrões de consumo e produção) e os recursos naturais renováveis e não renováveis. Podem envolver também questões ambientais globais (i.e., mudança climática, buraco na camada de ozônio); qualidade ambiental do ar, solo e água; globalização e qualidade das instituições.

Neste sentido, Becker et al. (2017) argumentam que índices de desempenho forçam instituições e governos a questionarem seus padrões e atuam como "forças motrizes" para a mudança no comportamento. 
A construção de índices de desempenho exige a definição de indicadores e deve também considerar as necessidades e expectativas dos países desenvolvidos e em desenvolvimento.

As Nações Unidas adotaram um novo conjunto de metas e indicadores com 17 Objetivos de Desenvolvimento Sustentável (ODS), para ser cumprido até 2030, com o propósito de acabar com todas as formas de pobreza, lutar contra as desigualdades e combater as alterações climáticas (ONU, 2015). É importante que os indicadores escolhidos reflitam não apenas o conhecimento das instituições governamentais, mas também as expectativas da sociedade. Tasaki et al. (2015) reforçam a necessidade de ter informações disponíveis e a promoção de um diálogo contínuo com a sociedade para a construção de índices de desempenho, alinhados com as particularidades de um país ou região.

As interligações entre aspectos ambientais, econômicos e sociais são difíceis de serem capturadas e refletidas nas medições e tendências ao longo do tempo (COOK et al., 2017). Para Moldan et al. (2012), o valor absoluto do índice (ou indicador) não é tão importante quanto a possibilidade de comparar os resultados alcançados. Apesar de os países apresentarem certa similaridade, não é possível afirmar qual o melhor modelo de desenvolvimento sustentável. De acordo com Cornescu et al. (2014), existem diferenças de localização geográficas, de recursos naturais, de governança e de políticas econômicas que impactam as metas a serem alcançadas.

\section{METODOLOGIA}

\section{Área de estudo}

A mesorregião do Nordeste Paraense, composta por 49 municípios e com uma área de 83.316,02 $\mathrm{Km}^{2}$, é considerada a mais antiga fronteira de colonização do estado do Pará, tendo se constituído em importante centro no abastecimento de alimentos, fibras e insumos energéticos da economia paraense desde o terceiro quartel do século XIX (REBELLO et al., 2017). Devido essa antiga ocupação, atualmente apenas 35\% dessa região é coberta por matas primárias originais (CORDEIRO et al., 2017). Segundo o IBGE essa mesorregião possui uma população, estimada para 2016, de 1.942.216 habitantes, com densidade demográfica de 23,31 hab/ $\mathrm{km}^{2}$.

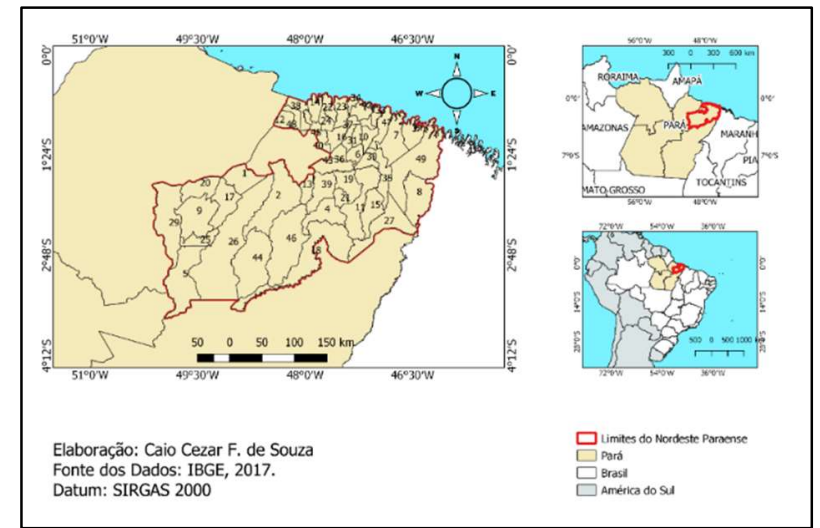

Figura 1: Delimitação geográfica do Nordeste Paraense no Estado do Pará. ${ }^{1}$

\footnotetext{
${ }^{1}$ Nota: (1) Abaetetuba, (2) Acará, (3) Augusto Corrêa, (4) Aurora do Pará, (5) Baião, (6) Bonito, (7) Bragança, (8) Cachoeira do Piriá, (9) Cametá, (10) Capanema, (11) Capitão Poço, (12) Colares, (13) Concórdia do Pará, (14), Curuçá, (15) Garrafão do Norte, (16) Igarapé-Açu, (17) Igarapé-Miri, (18) 
Apesar da crescente urbanização, o Nordeste Paraense ainda se mantém com características rurais, tendo 51,2\% da população vivendo no meio rural. Os setores de serviços e agropecuário são os que possuem o maior dinamismo econômico nessa mesorregião, representando 57,93\% e 34,29\% respectivamente do Valor Adicionado a preços correntes em 2013. Em relação a agropecuária pode-se destacar o cultivo da mandioca e do dendê, com áreas cultivadas de 151.190 ha e 65.515 ha respectivamente em 2014 (PEREIRA, 2017).

\section{Índice de desenvolvimento sustentável para municípios (IDSM)}

Para avaliar a sustentabilidade dos 49 municípios da mesorregião do Nordeste Paraense utilizou-se o Índice de Desenvolvimento Sustentável para Municípios (IDSM), conforme descrito por Martins et al. (2012). Porém, de modo a adaptar à realidade local e levando em consideração as informações levantadas em consultas às bases de dados disponíveis realizou-se um ajuste das dimensões e suas respectivas variáveis utilizadas por esses autores. Sendo assim, foram definidas as seguintes dimensões e seus respectivos indicadores, conforme apresentado no Quadro 1.

Quadro 1: Dimensões e Indicadores de Sustentabilidade adotados na pesquisa.

\begin{tabular}{|l|l|}
\hline DIMENSÃO & INDICADORES \\
\hline Dimensão Social & $\begin{array}{l}\text { Esperança de vida ao nascer/ Mortalidade Infantil/ Prevalência da desnutrição/ Imunização infantil/ } \\
\text { Ofertas de serviços básico de saúde/ Escolarização/ Qualidade do aprendizado do ensino básico/ } \\
\text { Escolaridade/ Analfabetismo/ Famílias atendidas com programas sociais/ Condição de moradia/ Taxa de } \\
\text { homicídio/ Taxa de mortes por acidente de trânsito. }\end{array}$ \\
\hline $\begin{array}{l}\text { Dimensão } \\
\text { Demográfica }\end{array}$ & $\begin{array}{l}\text { Crescimento da população/ Razão entre a população urbana e rural/ Densidade Demográfica/ Razão entre } \\
\text { a população masculina e feminina/ Razão de dependência. }\end{array}$ \\
\hline $\begin{array}{l}\text { Dimensão } \\
\text { Econômica }\end{array}$ & $\begin{array}{l}\text { PIB per capita/ Participação da indústria no PIB/ Saldo da balança comercial/ Renda per capita/ Índice } \\
\text { GINI/ Remuneração média do trabalhador formal. }\end{array}$ \\
\hline $\begin{array}{l}\text { Dimensão Político- } \\
\text { Institucional }\end{array}$ & $\begin{array}{l}\text { Acesso a telefonia fixa/ Despesas por função: administração, assistência social, saúde, educação, cultura, } \\
\text { urbanismo, saneamento, gestão ambiental, agricultura, comércio e serviços, energia, transporte/ } \\
\text { Participação nas eleições/ Fundo de participação dos municípios. }\end{array}$ \\
\hline Dimensão Ambiental & $\begin{array}{l}\text { Desflorestamento acumulado/ Acesso ao sistema de abastecimento de água/ Tipo de esgotamento } \\
\text { sanitário por domicílio/ Acesso a coleta de lixo urbana e rural/ Consumo médio per capita de água/ } \\
\text { Arborização de vias públicas. }\end{array}$ \\
\hline Dimensão Cultural & $\begin{array}{l}\text { Quantidade de bibliotecas, museus, unidades de ensino superior, estádios ou ginásios poliesportivos, } \\
\text { centros culturais e pontos de cultura. }\end{array}$ \\
\hline
\end{tabular}

Fonte: Adaptado de Martins et al. (2012).

Seguindo a metodologia descrita em Martins et al. (2012), em razão dos indicadores apresentarem diferentes unidades de medidas, transformou-se os mesmos em índices, cujo valor mínimo é 0 (zero) e o valor máximo é 1 (um). Para essa transformação foi considerado que esses indicadores podem ter relação positiva (quanto maior o indicador melhor será o índice) ou negativa (quanto maior o indicador pior será o índice), com isso após identificar a relação de cada variável foram utilizadas as seguintes fórmulas para o cálculo do índice:

$$
\begin{aligned}
& \text { Quando a relação é positiva: } I=(x-m) /(M-m) \\
& \text { Quando a relação é negativa: } I=(M-x) /(M-m)
\end{aligned}
$$

Ipixuna do Pará, (19) Irituia, (20) Limoeiro do Ajuru, (21) Mãe do Rio, (22) Magalhães Barata, (23) Maracanã, (24) Marapanim, (25) Mocajuba, (26) Moju, (27) Nova Esperança do Piriá, (28) Nova Timboteua, (29) Oeiras do Pará, (30) Ourém, (31) Peixe-Boi, (32) Primavera, (33) Quatipuru, (34) Salinópolis, (35) Santa Luzia do Pará, (36) Santa Maria do Pará, (37) Santarém Novo, (38) São Caetano de Odivelas, (39) São Domingos do Capim, (40) São Francisco do Pará, (41) São João da Ponta, (42) São João de Pirabas, (43) São Miguel do Guamá, (44) Tailândia, (45) Terra Alta, (46) Tomé-Açu, (47) Tracuateua, (48) Vigia, (49) Viseu. 
I = índice calculado para cada município analisado; $\mathrm{x}=$ valor de cada indicador em cada município; $\mathrm{m}=$ valor mínimo identificado nos municípios analisados; $M=$ valor máximo identificado nos municípios analisados.

Os dados referentes aos municípios do Nordeste Paraense foram obtidos pela coleta de dados secundários em diversas bases de dados, nos sites do Atlas do Desenvolvimento Humano no Brasil, Fundação Amazônia de Amparo a Estudos e Pesquisas (FAPESPA), Caderno de Informações de Saúde do DATASUS, Instituto Brasileiro de Geografia e Estatística (IBGE), Finanças do Brasil da Secretaria do Tesouro Nacional (FINBRA), Agência Nacional de Telecomunicações (ANATEL), Tribunal Superior Eleitoral (TSE) e Sistema Nacional de Informações sobre Saneamento (SNIS). Foram utilizados os dados mais atuais disponíveis em cada base.

Após a padronização dos dados, com a transformação em índices, realizou-se a agregação dos mesmos por dimensão através da média aritmética, obtendo-se assim os IDSM social, IDSM demográfico, IDSM econômico, IDSM político-institucional, IDSM ambiental e IDSM cultural. O IDSM da mesorregião do Nordeste Paraense foi alcançado através da determinação da média aritmética dos IDSMs das seis dimensões. Para traduzir os resultados obtidos pelo cálculo dos índices utilizou-se uma escala de níveis de sustentabilidade, ilustrada na Tabela 1, e em seguida foram elaborados mapas com a utilização do software free QGIS 3.0 para a melhor exposição desses resultados.

Tabela 1: Classificação e representação dos índices em níveis de sustentabilidade.

\begin{tabular}{|l|l|l|}
\hline ÍNDICE (0-1) & COLORAÇÃO & NÍVEL DE SUSTENTABILIDADE \\
\hline $1,0000-0,7501$ & & IDEAL \\
\hline $0,7500-0,5001$ & & ACEITÁVEL \\
\hline $0,5000-0,2501$ & & ALERTA \\
\hline $0,2500-0,0000$ & & CRÍTICO \\
\hline
\end{tabular}

Fonte: Adaptado de Martins et al. (2012).

\section{RESULTADOS E DISCUSSÃO}

A partir dos dados foi possível observar que cada indicador e município contribuíram de forma positiva ou negativa para o nível de sustentabilidade de cada dimensão analisada. Nesse sentido pode-se destacar que os indicadores de prevalência de desnutrição, escolarização, acesso ao sistema de abastecimento de água e consumo médio per capita de água foram os que apresentaram os maiores índices na média. Já os indicadores de quantidade de bibliotecas, museus, unidades de ensino superior, pontos de cultura, ginásios/estádios e centros culturais, acesso a telefonia fixa, tipo de esgotamento sanitário por domicílio, saldo da balança comercial, participação da indústria no PIB e oferta de serviços básicos de saúde foram os que apresentaram os menores índices na média. Também vale ressaltar que os municípios de Abaetetuba, Capanema e Salinópolis foram os que apresentaram os maiores índices na média e Cachoeira do Piriá, Nova Esperança do Piriá, Oeiras do Pará, São Domingos do Capim e Viseu foram os que apresentaram os menores índices na média. Logo, considerando as dimensões e os níveis de sustentabilidade apresentados, foram obtidos os resultados apresentados na Tabela 2. 
Tabela 2: Percentual dos níveis de sustentabilidade por dimensão.

\begin{tabular}{|c|c|c|c|c|c|c|c|}
\hline \multirow{2}{*}{$\begin{array}{l}\text { Nível } \\
\text { Sustentabilidade }\end{array}$} & \multicolumn{6}{|c|}{ Percentual por dimensão (\%) } & \multirow{2}{*}{$\begin{array}{l}\text { Percentual Nordeste } \\
\text { Paraense (\%) } \\
\text { Nordeste Paraense }\end{array}$} \\
\hline & Social & Demográfica & Econômica & $\begin{array}{l}\text { Político- } \\
\text { Institucional }\end{array}$ & Ambiental & Cultural & \\
\hline Ideal & 4 & 0 & 0 & 0 & 2 & 0 & 0 \\
\hline Aceitável & 78 & 61 & 6 & 10 & 67 & 0 & 6 \\
\hline Alerta & 18 & 39 & 71 & 78 & 31 & 20 & 94 \\
\hline Crítico & 0 & 0 & 23 & 12 & 0 & 80 & 0 \\
\hline Total & 100 & 100 & 100 & 100 & 100 & 100 & 100 \\
\hline
\end{tabular}

Na dimensão social 78\% dos municípios foram classificados com nível de sustentabilidade aceitável, o que indica a possibilidade da mesorregião analisada alcançar o nível ideal, haja vista também a inexistência de municípios em situação considerada crítica. Porém vale destacar que a metodologia utilizada faz uma comparação somente dentro da área analisada, ou seja, esses municípios apresentam uma situação aceitável levando em consideração a situação do Nordeste Paraense, que não pode ser considerado um padrão alto uma vez que o Estado do Pará, segundo o Atlas do Desenvolvimento Humano no Brasil tem o 4ㅇ pior IDH do Brasil. Também é importante ressaltar que no indicador de oferta de serviços básicos de saúde, que apresentou pior índice na dimensão social, apenas 6 municípios apresentaram condições piores que a média do estado, então infere-se que esse problema não está limitado apenas ao Nordeste Paraense.

Quanto a educação constatou-se que se teve maior abrangência do serviço nos últimos anos, pois se teve um alto índice de escolarização (crianças de 6 a 14 anos) e um baixo índice de escolaridade (população de 18 anos ou mais com ensino fundamental completo), porém a qualidade do ensino ainda deixa a desejar, uma vez que a média das notas do IDEB para os anos iniciais e finais do ensino fundamental foram de 3,9 e 3,4 respectivamente, enquanto que as médias nacionais foram de 5,5 e 4,5.

Em relação a dimensão demográfica, assim como na social, a maior parcela dos municípios apresenta o nível aceitável, entretanto o quantitativo de identificados em alerta é expressivo, isso pode ser explicado pela grande desigualdade na relação entre população urbana e rural entre os municípios, como exemplo se pode citar as cidades de Salinópolis e São João da Ponta que apresentam 89,2\% e 19,6\% de seus habitantes na zona urbana respectivamente e também o desequilíbrio na ocupação do espaço, que tem uma densidade demográfica média de 36,3 hab/ $\mathrm{km}^{2}$, mas tem o município de Salinópolis com 166,4 hab/km² e Nova Esperança do Piriá com 7,4 hab./km².

No tocante a dimensão econômica $94 \%$ dos municípios estão classificados nos menores níveis de sustentabilidade, alerta e crítico, com $71 \%$ e $23 \%$ respectivamente. Porém esse resultado pode não representar a realidade local, pois segundo Pereira (2017) o setor de serviços e agropecuário são os de maior importância econômica na região e o índice de participação das indústrias no PIB foi um dos que apresentaram menor valor nessa dimensão, demonstrando assim que o Nordeste Paraense apresenta pouco dinamismo econômico no setor industrial, tendo apenas os municípios de Ipixuna do Pará e Capanema mais de $20 \%$ de participação industrial no PIB, podendo destacar a indústria de cimento no último. Outro indicador que apresentou índice muito baixo foi o saldo da balança comercial, isso pode ser explicado pelo fato dos municípios estudados terem sua produção voltada, prioritariamente, para o abastecimento do mercado interno, tendo apenas os municípios de Abaetetuba, Bragança, Moju e Tailândia valores significativos de 
exportação, sendo a primeira devido à proximidade com as indústrias de alumínio de Barcarena, Bragança por ser o polo pesqueiro no Nordeste Paraense e os dois últimos devido as instalações das grandes empresas de exploração de dendê.

Os resultados da dimensão político-institucional evidenciam a situação de alerta, pois $78 \%$ dos municípios analisados estão agrupados nesse nível. Esse cenário está relacionado principalmente aos indicadores de acesso a telefonia fixa e fundo de participação dos municípios, esse está vinculado a desigualdade na distribuição de recursos da União, comparando as transferências e o número de habitantes das cidades e aquele está atrelado a disseminação da telefonia móvel o que leva muitos usuários a deixarem de usar os serviços de telefonia fixa.

Na dimensão ambiental se tem a distribuição dos municípios majoritariamente entre os níveis aceitável e alerta, com $67 \%$ e $31 \%$ respectivamente. O acesso ao abastecimento de água é o principal responsável por essa grande parte no nível aceitável, pois segundo o SNIS todas as cidades têm cobertura integral desse serviço, o que não quer dizer que todas têm acesso a água de qualidade, visto que é considerado os diversos tipos de abastecimento, não apenas das empresas responsáveis pelo sistema de abastecimento. Já o percentual em alerta está relacionado ao esgotamento sanitário inadequado, fato recorrente no Estado como um todo, uma vez que segundo o Instituto Trata Brasil (2018) no ranking do saneamento das 100 maiores cidades do Brasil o Pará aparece em três das quatro últimas posições com os municípios de Santarém, Belém e Ananindeua. Outro ponto a destacar negativamente nessa dimensão é alta taxa de desflorestamento acumulado, devido a essa região ter sido a mais antiga fronteira de colonização do Estado, o que levou ao desmatamento de diversas áreas para a expansão agrícola.

Finalmente, na dimensão cultural $80 \%$ dos municípios analisados apresentaram situação crítica. Porém esse resultado está vinculado a falta de equipamentos culturais, que foram os indicadores utilizados nessa dimensão, ou seja, na maioria das cidades é precária a quantidade de bibliotecas, museus, unidades de ensino superior, estádios ou ginásios poliesportivos e centros culturais, não estando, portanto, relacionado a falta de patrimônios histórico-cultural na mesorregião do Nordeste Paraense, visto que municípios como Bragança, possui diversas construções históricas e eventos como a Marujada tradicional manifestação cultural em honraria a São Benedito; Marapanim, que é considerada como 'Terra do Carimbó', ritmo musical considerado Patrimônio Cultural Imaterial do Brasil; e, Vigia que tem o Círio mais antigo do Pará e patrimônios históricos como a Igreja de Pedras.

Todos esses resultados por dimensão de sustentabilidade também foram ilustrados em mapas, para melhor visualização da situação da mesorregião do Nordeste Paraense, como pode ser observado na Figura 2. Assim como foram elaborados mapas dos níveis de sustentabilidade por dimensão, também foi gerado o mapa do IDSM geral da mesorregião do Nordeste Paraense, ilustrado na Figura 3. 


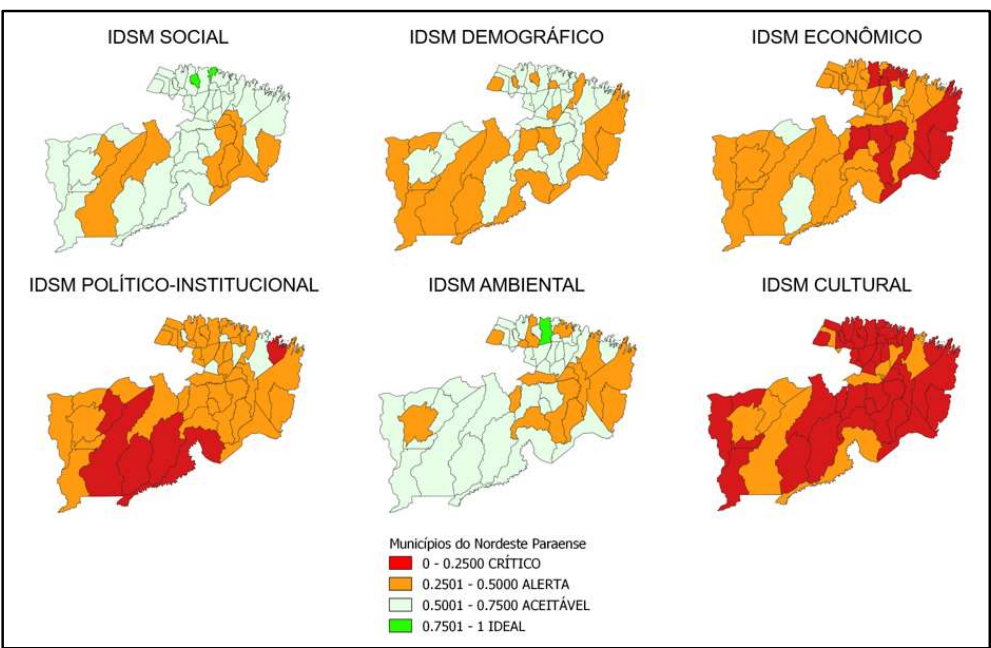

Figura 2: Mapa dos IDSMs por dimensão de sustentabilidade.

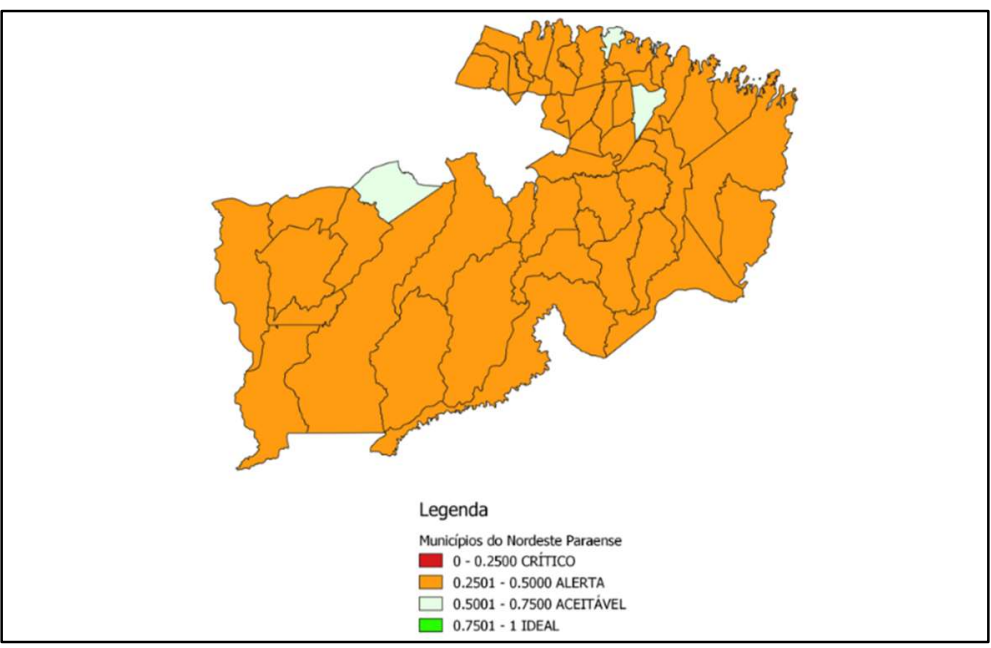

Figura 3: Mapa do IDSM dos municípios da Mesorregião do Nordeste Paraense.

Os resultados mostraram que apenas 6\%, ou seja, 3 dos 49 municípios (Abaetetuba, Capanema e Salinópolis) apresentaram nível aceitável. Demonstrando assim que a partir do que foi observado para cada dimensão estas cidades são as que apresentaram melhor estrutura em comparação com as demais do Nordeste Paraense, porém não se pode afirmar que as mesmas apresentam o mesmo nível se comparadas em uma escala maior, como estadual ou nacional.

Cabe o registro que esses três municípios têm seu processo de urbanização influenciado por aspectos de seu relativo dinamismo. No caso de Abaetetuba pela função de entreposto comercial para os municípios do entorno da área de influência do polo de Barcarena, tendo se destacado historicamente com o tradicional comércio de regatão, que ajudou a consolidar, inclusive, grupos empresariais fortes no setor supermercadista paraense. Capanema em relação a sua função de nó rodoviário e as oportunidades no comércio e na indústria de cimento. Salinópolis em razão, principalmente, da consolidação do polo turístico e do vultoso investimento em infraestrutura que se deu em função disso.

\section{CONCLUSÕES}

A metodologia utilizada, Índice de Desenvolvimento Sustentável para Municípios, evidenciou o estado de alerta nos municípios da mesorregião do Nordeste Paraense, destacando as dimensões cultural, 
econômica e político-institucional que apresentaram a maior quantidade de cidades nos níveis alerta e crítico.

Os resultados obtidos podem ser utilizados para orientar, principalmente, os gestores públicos na formulação de políticas mais eficientes que consigam estabelecer um equilíbrio entre o crescimento econômico, a preservação ambiental e o suporte às necessidades básicas da população. Na mesorregião analisada a qualidade da educação, oferta de serviços básicos de saúde, incentivo ao setor industrial, saneamento básico e a construção de equipamentos culturais foram identificados como os principais pontos a serem abordados por essas políticas públicas.

Portanto, o IDSM se mostrou uma ferramenta válida para avaliação da sustentabilidade em municípios, sendo que os indicadores podem ser adaptados de acordo com cada localidade, a fim de proporcionar o melhor resultado.

\section{REFERÊNCIAS}

AYRES, R. U.. Sustainability economics: Where do we stand?. Ecological Economics, v.67, n.2, p.281-310, 2008. DOI: https://doi.org/10.1016/j.ecolecon.2007.12.009

BARTELMUS, P.. Dematerialization and capital maintenance: two sides of the sustainability coin. Ecological Economics, v.46, n.1, p.61-81, 2003. DOI: https://doi.org/10.1016/S0921-8009(03)00078-8

BECKER, W.; SAISANA, M.; PARUOLO, P.; VANDECASTEELE, I. Weights and importance in composite indicators: closing the gap. Ecological Indicators, v.80, p.12-22, 2017. DOI: https://doi.org/10.1016/j.ecolind.2017.03.056

BELLEN, H. M. V.. Indicadores de sustentabilidade: uma análise comparativa. 2 ed. Rio de Janeiro: Fundação Getúlio Vargas, 2006.

CMMAD. Comissão Mundial sobre Meio Ambiente e Desenvolvimento. Nosso futuro comum. Rio de Janeiro: Editora da Fundação Getúlio Vargas, 1991.

COOK D.; SAVIOLIDIS, N. M.; DAVIDSDOTTIR, B.; JOHANNSDOTTIR, L.; OLAFSSON, S.. Measuring countrie's environmental sustainability performance: the development of a nation-specific indicator set. Ecological Indicators, v.74, p.463-478, 2017. DOI:

https://doi.org/10.1016/j.ecolind.2016.12.009

CORDEIRO, I. M. C. C.; ARBAGE, M. J. C.; SCHWARTZ, G. Nordeste do Pará: configuração atual e aspectos indenitários. In: CORDEIRO, I. M. C. C.; RANGELVASCONCELOS, L. G. T.; SCHWARTZ, G.; OLIVEIRA, F. A.. Nordeste Paraense: panorama geral e uso sustentável das florestas secundárias. Belém: EDUFRA, 2017.

CORNESCU, V.; ADAM, R.. Considerations regarding the role of indicators used in the analysis and assessment of sustainable development in the E.U. Procedia Economics and Finance, v.8, p.10-16, 2014. DOI:

https://doi.org/10.1016/S2212-5671(14)00056-2

DAHL, A. L.. The Big Picture: Comprehensive approaches: Part one: Introduction. In: MOLDAN, B.; BILLHARZ, S. .
Sustainability indicators: A report on the project on indicators of sustainable development. Chichester: John Wiley and Sons, 1997.

ELKINGTON, J.. Towards the sustainable corporation: Winwin-win business strategies for sustainable development. California Management Review, v.36, n.2, p.90-100, 1994. DOI: https://doi.org/10.2307/41165746

GALLOPIN, G. C.. Environmental and sustainability indicators and the concept of situational indicators. A system approach. Environmental Modelling \& Assessment, v.1, p.101-117, 1996. DOI: https://doi.org/10.1007/BF01874899

GUIMARÃES, R. P.. Aterrizando una Cometa: indicadores territoriales de sustentabilidad. Santiago: ILPES, 1998.

HART, S. L.. Guide to sustainable community indicators. North Andover: Hart Environmental Data, 1999.

INSTITUTO TRATA BRASIL. Ranking do saneamento. São Paulo: GO Associados, 2018.

KATES, R. W.; CLARK, W. C.; CORELL, R.; HALL, J. M.; JAEGER, C. C.; LOWE, I.; MCCARTHY, J. J.; SCHELLNHUBER, H. J.; BOLIN, B.; DICKSON, N. M.; FAUCHEUX, S.; GALLOPIN, G. C.; GRUBLER, A.; HUNTLEY, B.; JAGER, J.; JODHA, N. S.; KASPERSON, R. E.; MABOGUNJE, A.; MATSON, P.; MOONEY H.; MOORE, B.; O'RIORDAN, T.; SVEDIN, U.. Sustainability science. Science, v.292, n.5517, p.641-642, 2001. DOI: https://doi.org/10.1126/science.1059386

LEHTONEN, M.. The environmental-social interface of sustainable development: capabilities, social capital, institutions. Ecological Economics, v.49, n.2, p.199-214, 2004. DOI: https://doi.org/10.1016/j.ecolecon.2004.03.019

LOZANO, R.. Towards better embedding sustainability into companies' systems: an analysis of voluntary corporate initiatives. Journal of Cleaner Production, v.25, p.14-26, 2012. DOI: https://doi.org/10.1016/j.jclepro.2011.11.060

MARTINS, M. F.; CÂNDIDO, G. A.. Índice de desenvolvimento sustentável para localidades: uma proposta metodológica de 
construção e análise. Revista de Gestão Social e Ambiental, São Paulo, v.6, n.1, p.03-19, 2012. DOI:

https://doi.org/10.24857/rgsa.v6i1

MASERA, O.; ASTIER, M.; LÓPEZ-RIDAURA, S.. Sustentabilidad y manejo de recursos naturales: el marco de evaluación MESMIS. Ciudad de México: Mundi-Prensa, 1999.

MOLDAN, B.; JANOUŠKOVÁ, S.; HÁK, T.. How to understand and measure environmental sustainability: indicators and targets. Ecological Indicators, v.17, p.4-13, 2012. DOI: https://doi.org/10.1016/j.ecolind.2011.04.033

MOURA, L. G. V.; ALMEIDA, J.; MIGUEL, L. A.. Avaliação de sustentabilidade em agroecossistemas: um pouco de pragmatismo. REDES, v.9, n.2, p.133-155, 2004.

ONU. Transformando nosso mundo: a agenda 2030 para o desenvolvimento sustentável. Nova York: Nações Unidas, 2015.

PEREIRA, C. L. O.. Abordagem socioeconômica da mesorregião Nordeste do Pará. In: CORDEIRO, I. M. C. C.; RANGEL-VASCONCELOS, L. G. T.; SCHWARTZ, G.; OLIVEIRA, F. A.. Nordeste Paraense: panorama geral e uso sustentável das florestas secundárias. Belém: EDUFRA, 2017.

QUIROGA, R.. Indicadores de sostenibilidad ambiental y de desarrollo sostenible: estado del arte y perspectivas. Santiago de Chile: CEPAL, 2001.
REBELLO, F. K.; HOMMA, A. K. O.. História da colonização do Nordeste Paraense: uma reflexão para o futuro da Amazônia. Belém: EDUFRA, 2017.

REZENDE, G. B. M.; CÂNDIDO, G. A.; REZENDE, H. L.; SILVA, F. P.. Sustentabilidade de Barra do Garças sob a Ótica do Índice de Desenvolvimento Sustentável para Municípios.

Desenvolvimento em questão, v.15, n.39, p.203-235, 2017. DOI: https://doi.org/10.21527/2237-6453.2017.39.203-235

SALAS-ZAPATA, W.; RÍOS-OSORIO, L.; CASTILLO, J. A. D.. La ciencia emergente de la sustentabilidad: de la práctica científica hacia la constitución de una ciencia. Interciencia, v.36, n.9, p.699-706, 2011.

SEGNESTAM, L.. Indicators of environment and sustainable development: theories and practical experience. Washington: World Bank, 2002.

SIENA, O.. Método para avaliar progresso em direção ao desenvolvimento sustentável. Tese (Doutorado em Engenharia de Produção) - Universidade Federal de Santa Catarina, Florianópolis, 2002.

TASAKI, T.; KAMEYANA, Y.. Sustainability indicators: are we measuring what we ought to measure? Global Environmental Research, v.19, p.147-154, 2015.

A CBPC - Companhia Brasileira de Produção Científica (CNPJ: 11.221.422/0001-03) detém os direitos materiais desta publicação. Os direitos referem-se à publicação do trabalho em qualquer parte do mundo, incluindo os direitos às renovaç̃ões, expansões e disseminações da contribuiç̃o, bem como outros direitos subsidiários. Todos os trabalhos publicados eletronicamente poderão posteriormente ser publicados em coletâneas impressas sob coordenação da Sustenere Publishing, da Companhia Brasileira de Produção Científica e seus parceiros autorizados. Os (as) autores (as) preservam os direitos autorais, mas não têm permissão para a publicação da contribuição em outro meio, impresso ou digital, em português ou em tradução. 